\title{
Acute Myocardial Infarction Due to Spontaneous Coronary Artery Dissection - A Series of Five Cases
}

\author{
Roberto Ramos Barbosa ${ }^{1}$, Fábio Salerno Rinaldi², J. Ribamar Costa Jr. ${ }^{3}$, Fausto Feres ${ }^{4}$, \\ Alexandre Abizaid ${ }^{5}$, Amanda G. M. R. Sousa ${ }^{6}$, J. Eduardo Sousa ${ }^{7}$
}

\begin{abstract}
We report a series of five patients hospitalized due to acute coronary syndrome, from 2008 to 2010, in whom coronary angiography showed spontaneous coronary dissection. Spontaneous coronary artery dissection is a rare cause of acute myocardial infarction, most commonly seen in young individuals with no history of cardiovascular disease. The optimal management is uncertain, especially due to the limited clinical experience with this entity. Intravascular ultrasound is a useful tool to diagnose spontaneous coronary artery dissection as well as to guide percutaneous therapy.
\end{abstract}

DESCRIPTORS: Acute coronary syndrome. Myocardial infarction. Coronary vessels. Angioplasty. Stents.

$S$ pontaneous coronary dissection is a rare entity, usually found in the literature as isolated case reports, which generally affects young and/or female patients. ${ }^{1}$ In most cases, classical risk factors for coronary atherosclerotic disease (CAD) are absent. Since it is an infrequently studied disease, its etiology remains largely unknown. It can cause death in up to $40 \%$ of cases, usually in the first days of the clinical picture. $^{2}$ The prognosis and the therapeutic approach remain uncertain.

Although it usually occurs in arteries without obstructive atherosclerotic disease at the angiography,
RESUMO

\author{
Infarto Agudo do Miocárdio por \\ Dissecção Espontânea de Artérias \\ Coronárias - Série de Cinco Casos
}

Relatamos série de 5 pacientes internados por síndrome coronária aguda, entre 2008 e 2010, nos quais a cinecoronariografia evidenciou dissecção coronária espontânea. A dissecção coronária espontânea é uma causa rara de infarto do miocárdio, mais comumente observada em indivíduos jovens e sem histórico de doenças cardiovasculares. O manejo ideal é incerto, principalmente pela limitada experiência clínica com essa entidade. A ultrassonografia intracoronária é uma ferramenta útil tanto para o diagnóstico da dissecção coronária espontânea como para guiar o tratamento percutâneo.

DESCRITORES: Síndrome coronariana aguda. Infarto do miocárdio. Vasos coronários. Angioplastia. Stents.

spontaneous coronary dissection may be related with the inflammatory reaction in the medial layer of vessels, mainly initiated by autoantibodies produced during pregnancy or in the first weeks after childbirth, ${ }^{3}$ as a result of antiphospholipid antibody syndrome or changes in the extracellular matrix metabolism. ${ }^{4}$ Thus, an endothelial dysfunction appears to be the physiopathological basis of spontaneous coronary dissection.

The present study reports on a series of five cases of acute myocardial infarction (AMI) with or without ST-segment elevation (STEMI and NSTEMI patients, respectively), treated at the Instituto Dante Pazzanese

\footnotetext{
1 Mastership. Interventionist cardiologist physician of the Instituto de Cardiologia do Espírito Santo. Vitória, ES, Brazil.

2 Interventionist Cardiologis Physician of the Santa Casa de Marília. Marília, SP, Brazil.

3 Doctor. Interventionist cardiologist physician of the Interventional Cardiology Services of the Instituto Dante Pazzanese de Cardiologia. São Paulo, SP, Brazil.

${ }^{4}$ Doctor. Interventionist cardiologist physician of the Interventional Cardiology Services of the Instituto Dante Pazzanese de Cardiologia. São Paulo, SP, Brazil.

${ }^{5}$ Full professor. Director of the Interventional Cardiology Service of the Instituto Dante Pazzanese de Cardiologia. São Paulo, SP, Brazil.
}

\footnotetext{
${ }^{6}$ Full professor. Director general of the Instituto Dante Pazzanese de Cardiologia. São Paulo, SP, Brazil.

7 Full professor. Director of the Center of Interventions in Structual Heart Diseases of the Instituto Dante Pazzanese de Cardiologia. São Paulo, SP, Brazil.
}

Correspondence to: Roberto Ramos Barbosa. Av. Marechal Campos, 1.579 - Santa Cecília - Vitória, ES, Brazil - CEP 29043-260

E-mail:roberto.rb@cardiol.br

Received on: 01/29/2013 • Accepted on: 04/27/2013 
de Cardiologia between January of 2008 and February of 2011, in whom the coronary angiography showed or was suggestive of spontaneous coronary dissection.

\section{CASE REPORTS}

\section{Case 1}

Male patient, 41 years old, with no CAD risk factors, sought medical care due to typical angina chest pain at rest. Electrocardiogram (ECG) at admission showed ST elevation $<1 \mathrm{~mm}$ in the inferior wall. Subsequent ECGs did not evidence of alterations in relation to the admission ECG. There was mild elevation of myocardial necrosis markers. Myocardial perfusion scintigraphy was performed in the presence of chest pain and evidenced hypoperfusion, suggesting inferior wall ischemia (Figure 1A). The patient was submitted to invasive stratification six hours after hospital admission. Coronary angiography showed spontaneous dissection in the proximal third of the right coronary artery, with $70 \%$ stenosis at its most critical point (Figure 1B). The other coronary arteries showed no lesions. After contrast injection and image acquisition for the right coronary artery, the patient went into cardiorespiratory arrest in ventricular fibrillation, which was promptly reversed with biphasic defibrillation at $200 \mathrm{~J}$. There was slowing of the right coronary artery flow. It was decided to perform a percutaneous coronary intervention $(\mathrm{PCl})$ with a bare-metal stent to the right coronary artery (Figure $1 \mathrm{C}$ ), which was successful (Figure 1D). Follow-up of 34 months showed clinical evolution without adverse cardiac events. The
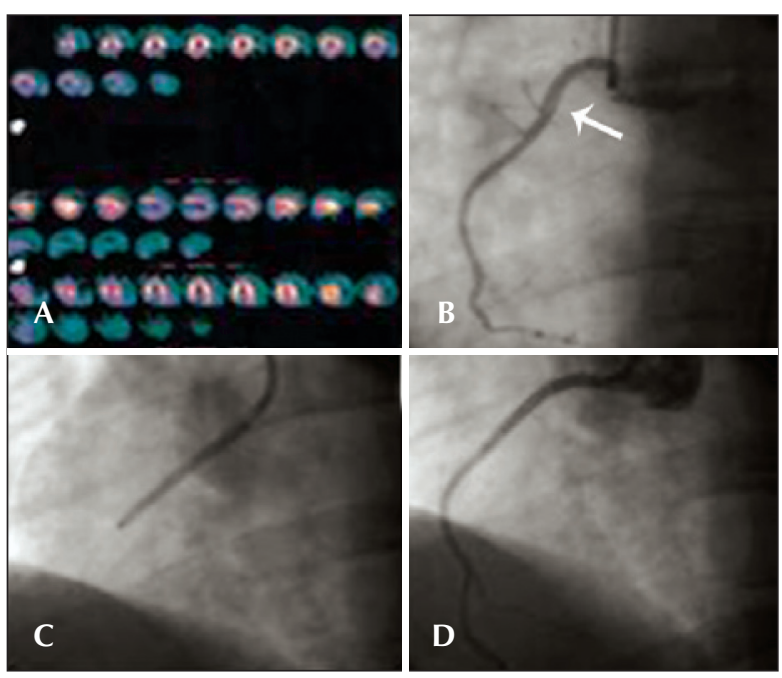

Figure 1 - In A, myocardial perfusion scintigraphy, performed in the presence of chest pain, shows hypoperfusion in the inferior wall. In $\mathrm{B}$, coronary angiography shows spontaneous dissection in the proximal third of the right coronary artery (arrow). In C, performance of percutaneous coronary intervention in the right coronary artery. In D, optimal angiographic result after percutaneous coronary intervention with stenting. patient is currently using acetylsalicylic acid (ASA) and simvastatin.

\section{Case 2}

Female patient, 37 years old, in postpartum period, without CAD risk factors, sought medical care presenting with typical chest pain, mild, which persisted hours after an episode of severe pain. Optimized clinical treatment was established, with complete pain relief. She was transferred to the Instituto Dante Pazzanese de Cardiologia with a diagnostic hypothesis of NSTEMI. She remained hemodynamically stable. The ECG showed a pathological $Q$ wave in the inferior wall, with no ST elevation (30 hours after the episode of severe chest pain). A coronary angiography was performed three days later and showed an image suggestive of spontaneous dissection in the middle third of the right coronary artery, without significant obstructive lesion, with normal distal flow (Figure 2A). The other coronaries showed no lesions; left ventriculography showed inferior-mid-basal wall akinesia. An intravascular ultrasound (IVUS) of the right coronary artery was performed, which confirmed the coronary dissection, showing an intimal flap (Figure $2 \mathrm{~B}$ ) and vessel wall hematoma (Figure 2C). Due to the patient's clinical stability, the prolonged evolution of the event (STEMI), and akinesia of the ventricular segment involved with no signs of viability, it was decided not to perform the $\mathrm{PCl}$, maintaining the clinical treatment. Follow-up of 29 months has shown evolution with no adverse cardiac events. The patient is currently using ASA, atenolol, and atorvastatin.

\section{Case 3}

Male patient, 34 years of age, with no risk factors for CAD, was admitted to another service presenting mild dyspnoea, approximately 24 hours after an episode of poorly characterized chest pain. He was transferred to the Instituto Dante Pazzanese de Cardiologia with a diagnosis of STEMI in anterior wall, with late presentation. He remained hemodynamically stable, without chest pain. ECG showed pathological Q wave in the extensive anterior wall, with ST elevation of $2 \mathrm{~mm}$ (six days after the episode of chest pain). Coronary angiography was performed eight days after the start of the clinical picture and presented image suggestive of
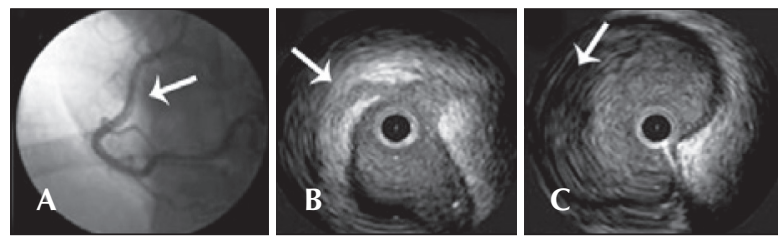

Figure 2 - In A, coronary angiography shows image suggestive of spontaneous dissection in the middle third of the right coronary artery (arrow), with normal distal flow. In B, intravascular ultrasound demonstrates intima flap (arrow). In C, intravascular ultrasound shows evidence of vessel wall hematoma (arrow). 
spontaneous dissection in the proximal third of the left anterior descending artery with moderate reduction in calibre in this segment and normal distal flow (Figure $3 \mathrm{~A})$. The other coronary arteries did not present any lesions. Left ventriculography demonstrated anterior wall akinesia and apical dyskinesia (Figure 3B). IVUS was not performed. Due to the patient's clinical stability, prolonged evolution of the event, and akinesia in the ventricular segment involved, it was decided not to perform $\mathrm{PCl}$, maintaining the clinical treatment. Clinical follow-up of 18 months showed no adverse cardiac events. Echocardiogram showed akinesia of the anterolateral wall and apical dyskinesia of the left ventricle, with left ventricular ejection fraction of $35 \%$. The patient is in functional class II and has not required hospitalisation for heart failure during this period. He is currently using carvedilol, enalapril, spironolactone, ASA, and simvastatin.

\section{Case 4}

Female patient, 24 years old, with no CAD risk factors, was admitted to the emergency room with typical chest pain, of strong intensity, accompanied by dizziness and nausea, radiating to the left arm, with duration of 2 hours. ECG showed ST segment elevation in the inferior wall. The patient was then referred to the catheterisation lab for possible primary $\mathrm{PCl}$. Coronary angiography evidenced sudden and regular narrowing in the transition from the proximal third to the middle third of the right coronary artery, and the distal segment of the coronary artery had reduced calibre, but maintained the epicardial flow (Figure 4A). There was no obstructive disease in the left coronary artery. At the end of the diagnostic procedure, the patient experienced significant pain relief. IVUS was performed for the right coronary artery, showing dual lumen of long extension, with evidence of hematoma in the false lumen (Figure $4 \mathrm{~A})$. Due to the patient's clinical stability and pain relief
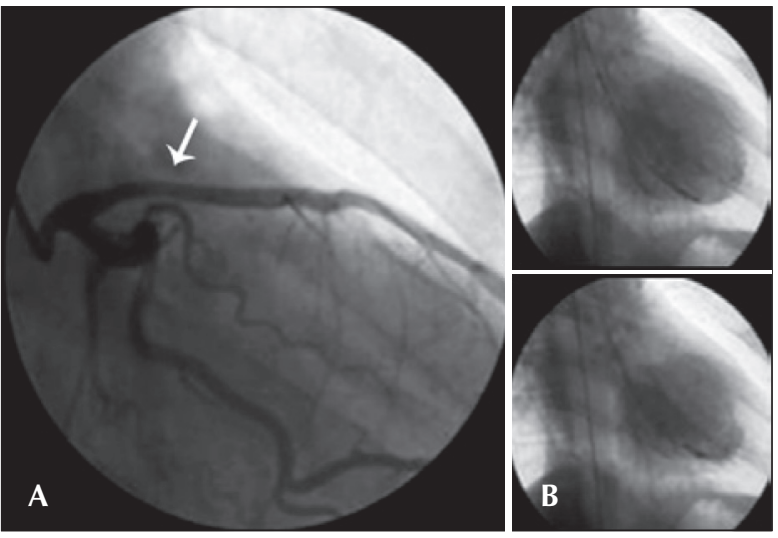

Figure 3. In A, coronary angiography shows spontaneous dissection in the proximal third of the left anterior descending artery (arrow). In B, Left ventriculography shows akinesis of the anterior wall and apical dyskinesia. at that moment, as well as the difficulty to define the extension of the coronary dissection, it was decided not to perform $\mathrm{PCl}$, and clinical treatment was maintained. Admission ECG at the coronary unit demonstrated that the ST segment returned to baseline levels and clinical outcome was satisfactory. One month after the event, the patient underwent computed tomography of the coronary arteries, which confirmed the resolution of the dissection and recovery of the lumen diameter throughout the right coronary artery (Figure 4B). Follow-up of 14 months has shown no adverse cardiac events, and the patient is currently using ASA.

\section{Case 5}

Female patient, 43 years old, smoker, was admitted with chest pain lasting for eight hours. ECG on admission showed inverted $\mathrm{T}$ wave in the anterior wall, as well as an increase of myocardial necrosis markers. Coronary angiography showed a clear image of spontaneous dissection in the left main coronary artery with extension of the dissection to the middle third of the left circumflex artery, with slowing of distal flow in this vessel. After contrast injection and image acquisition of left coronary artery, there was extension of the dissection to the left anterior descending artery, with $90 \%$ stenosis in its most critical point (Figure 5). There were no significant lesions in the right coronary artery. The patient remained hemodynamically stable, with mild chest pain. Intravenous nitroglycerin was immediately administered, and the cardiac surgical team was called. Emergency coronary artery bypass graft (CABG) surgery was performed on the same day, with placement of internal mammary artery graft to the left anterior descending artery and saphenous vein graft to the first marginal branch of the left circumflex artery. In the immediate postoperative period, there was bleeding increased by the mediastinal and left pleural drains, and surgical rapprochement was necessary to

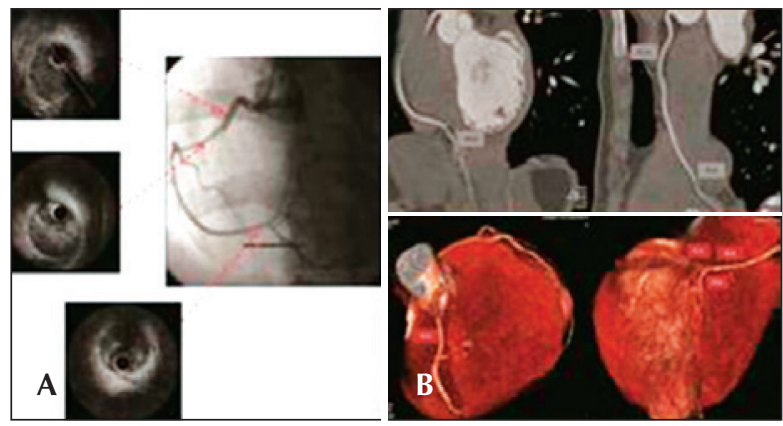

Figure 4 - In A, coronary angiography shows spontaneous dissection in the right coronary artery - sudden narrowing of the vessel in the transition of its proximal segment to medium. Intravascular ultrasound (IVUS) shows long extension dual lumen, with the presence of large hematoma in the false lumen. In B, coronary CT performed one month after the acute episode shows complete healing of the hematoma and restoration of the vascular lumen. 
control the bleeding. The patient remained in critical condition after reoperation, with difficulty weaning off of vasoactive drugs and worsening of shock, with ST elevation in the anterior wall; death occurred on the second postoperative day.

\section{DISCUSSION}

When treating a young patient with a diagnosis of AMI without risk factors for CAD and coronary angiography with no signs of severe obstructive lesions, it is imperative to consider the possibility of non-atherosclerotic coronary disease, such as coronary vasospasm, Takotsubo syndrome, and spontaneous coronary dissection. The latter, in turn, in spite of its little known causes, may be associated with pregnancy and postpartum autoimmune phenomena, antiphospholipid antibody syndrome, and fibromuscular dysplasia. ${ }^{5}$ However, since many cases lack the clinical identification of any of these factors, it is presumed that there is some endothelial dysfunction mechanism that is not fully understood, which leads to spontaneous coronary dissection.

The five cases described here illustrate the clinical presentation and diagnostic uncertainty involving spontaneous coronary dissection. The clinical presentation often occurs later, due to scepticism of both patients and family members, as well as of physicians, regarding the diagnosis of acute coronary syndrome (ACS). The low clinical risk of patients, who are generally young and do not have the classical risk factors for CAD, tends to delay the initial treatment and invasive treatments, such as coronary angiography.

Case 2, who was a postpartum patient, was submitted to antiphospholipid antibody syndrome test after

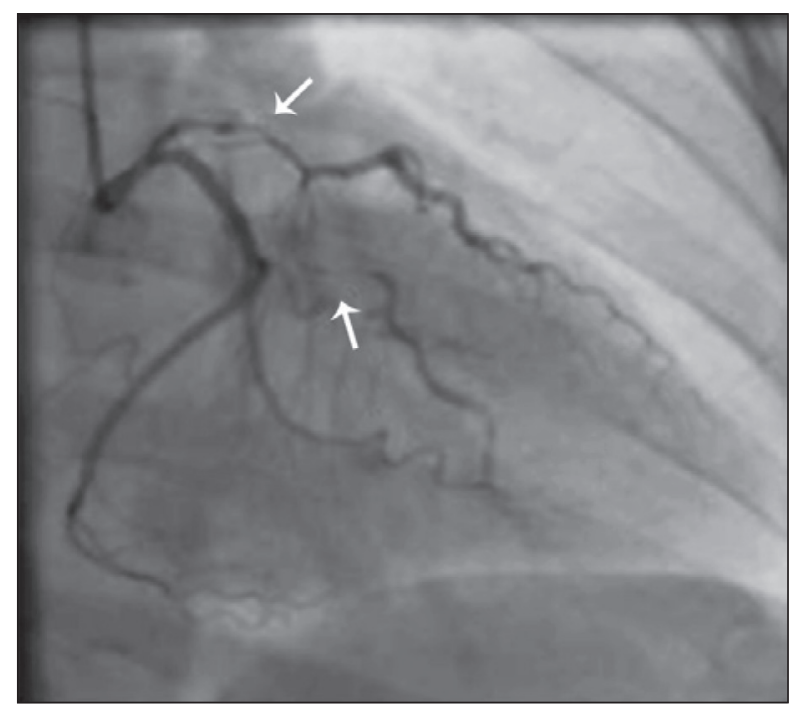

Figure 5 - Coronary angiography demonstrates spontaneous dissection in the left main coronary artery, with the dissection extending to the left anterior descending artery and left circumflex artery (arrows). hospital discharge, which was not confirmed, leaving a presumptive diagnosis of autoimmune phenomenon related to pregnancy and the postpartum period. Case 4 disclosed the use of anovulatory drug (oral contraceptive), which may or may not be related to the cardiac event; however, it was not possible to accurately establish a causal association between the medication and the spontaneous coronary dissection. In case 5, it is assumed that smoking contributed to endothelial dysfunction, culminating in spontaneous coronary dissection; however, in the absence of corroborative methods for this association, this hypothesis remains speculative. In the other cases, no disease or factor was clearly identified as a determinant of spontaneous coronary dissection in the acute phase of the event or during the subsequent clinical investigation. Thus, as commonly observed in these cases, no aetiology was confirmed by laboratory tests and associated with spontaneous coronary dissection in the reported cases, which would be of interest for therapeutic follow-up and for future studies.

All spectra of ACS may be present in the clinical presentation of spontaneous coronary artery dissection, including sudden death (up to $50 \%$ of cases, usually involving the left coronary trunk), in which the diagnosis in most cases is attained at the necropsy, with limited angiographic documentation. ${ }^{6}$ The clinical and angiographic diagnosis of this entity may be underestimated, since most cases do not undergo assessment through valuable diagnostic tools such as IVUS or optical coherence tomography, but instead undergo intervention in stenotic coronary lesions or simply have CAD ruled out in the absence of these lesions, without searching for an underlying cause for the presenting clinical picture. The angiographic appearance, despite suggestive, may not clearly disclose the coronary artery dissection, which must be considered when there is (together with the described clinical criteria) sudden and significant reduction in calibre, obstruction with smooth edges, and no spect of atherosclerotic disease in the coronary arteries or line of dissection with or without false lumen.

A condition most commonly found in women, spontaneous coronary dissection has a female to male ratio of $3: 1 ; 25 \%$ to $31 \%$ of cases are observed during pregnancy or the puerperal period. ${ }^{2}$ In females, dissections occur predominantly in the left coronary artery (87\%), generally affecting young women without traditional risk factors. Men, in turn, tend to be affected at an older age and, sometimes, with the presence of CAD risk factors, with involvement of the right coronary artery in $67 \%$ of cases. Generally, the left anterior descending artery is affected in $75 \%$ of cases; the right coronary artery in $20 \%$, the left circumflex artery in $4 \%$; and the left main coronary artery in less than $1 \%$ of cases. A strong association is observed between spontaneous coronary artery dissection and pregnancy, postpartum period, and oral contraceptive use. ${ }^{8}$ During pregnancy, pathological changes may occur in the arterial wall 
due to fragmentation of reticular fibres, hypertrophy of smooth muscle cells, and changes in the content of mucopolysaccharides and protein composition, leading to weakening of the vessel wall and, to its rupture during labor or afterwards. ${ }^{6}$

The vascular plane where the spontaneous dissection occurs most frequently is the outer third of the tunica media or between the media and adventitia. ${ }^{6}$ Histological findings in postmortem studies have shown inflammatory infiltrate, mainly eosinophilic, in the adventitial layer of vessels in $25 \%$ to $40 \%$ of patients who died from spontaneous coronary dissection. Proteases released by eosinophils may promote proteolytic reactions in the vascular walls, and may contribute to dissection.

Due to its rarity, the best treatment for spontaneous coronary dissection has not been determined, and may vary according to the clinical presentation, considering the persistence or relief of ischemic symptoms, the patient's hemodynamic status, coronary anatomy and dissection extension, the number of involved vessels, and the distal coronary flow. Although there is no robust scientific evidence, IVUS can be a valuable tool in cases of spontaneous coronary dissection, confirming diagnosis and measuring its length. ${ }^{9}$

In clinically-stable patients, especially when coronary blood flow is restored, a conservative medical approach may be considered, taking into account the high incidence of spontaneous healing of the dissection and low incidence of long-term adverse events. The use of nitrates and calcium-channel blockers can reduce local spasm and dissection progression; beta-blockers may be used to minimize hemodynamic forces, and antiplatelet drugs can prevent platelet aggregation and subsequent intramural thrombus formation. The use of fibrinolytics is controversial in this scenario - although the dissolution of intramural thrombus may appear, initially, as an attractive benefit, the use of these drugs can cause the propagation of the dissection. ${ }^{10}$ Buys et al. ${ }^{11}$ reported the case of a 31-year-old woman admitted with ACS caused by spontaneous coronary dissection, who showed, after the use of thrombolytic therapy, worsening of bleeding in the false lumen and extension of the dissection. The currently available data are not sufficient to define the role of antithrombotic agents and glycoprotein IIb/IIla inhibitors in these cases.

In clinically unstable patients or those with persistent ischemic symptoms, a more invasive approach should be considered, possibly with a reperfusion strategy, which is likely to provide better survival probability. $\mathrm{PCl}$ should be the first option in dissections involving a single vessel, especially when there is involvement of its entire length. It is often necessary to implant more than one stent to treat the dissection, and there is no proven benefit of drug-eluting stents when compared to bare-metal stents in such circumstances. Stent implantation is intended to seal the intimal flap to re-expand the true lumen. Special care should be used to minimize the risk of obstructing intramural hematomas present in the false lumen, since the elimination of the latter can compress the hematoma, dislocating it to the proximal or distal border of the stent, perpetuating the dissection. ${ }^{10}$ Furthermore, the possibility of having the guide in the false lumen should be completely discarded before expanding the stent. IVUS can be useful to clarify the situation, and its use is recommended to evaluate stent expansion and strut apposition in relation to the vessel walls, and also to prevent entry into the false lumen. ${ }^{9}$

CABG has high mortality in this scenario (up to $33 \%$ ), and should be reserved for dissections involving the left coronary trunk or multiple vessels. However, CABG can be technically difficult, as the dissection may extend further distally than shown by the angiography, complicating the decision regarding placement of the graft. Furthermore, the dissection can subsequently extend to the distal parts of the revascularized vessels, compromising the surgical outcome; ${ }^{6}$ this is the likely adverse event the patient in case 5 experienced, culminating with her death in the postoperative period.

Despite the recommendation to individualize the therapeutic strategy, the long-term results for survivors of spontaneous coronary dissection are generally encouraging, with low rates of event recurrence (either in the same vessel or another). However, the optimal method of follow-up for these patients is yet to be established, as is the best conduct to prevent further episodes. In general, it is concluded that spontaneous coronary dissection is an infrequent cause of ACS, which usually affects young individuals, and whose ideal management is uncertain, partially due to the limited experience with this clinical entity. IVUS is a useful diagnostic tool, especially in cases in which the dissection is not clearly visible at the coronary angiography, and can also guide and optimize percutaneous treatment. ${ }^{12}$ When there is hemodynamic or electrical instability, or persistent chest pain, a CABG strategy should be considered, given the severity of the usually observed clinical presentation.

\section{CONFLICTS OF INTEREST}

The authors declare no conflicts of interest.

\section{REFERENCES}

1. Kamineni R, Sadhu A, Alpert JS. Spontaneous coronary artery dissection: report of two cases and a 50-year review of the literature. Cardiol Rev. 2002;10(5):279-84.

2. Koller PT, Cliffe CM, Ridley DJ. Immunosuppressive therapy for peripartum-type spontaneous coronary artery dissection: case report and review. Clin Cardiol. 1998;21(1):40-6.

3. Bonnet J, Aumailley M, Thomas D, Grosgogeat Y, Broustet JP, Bricaud H. Spontaneous coronary artery dissection: case report and evidence for a defect in collagen metabolism. Eur Heart J. 1986;7(10):904-9.

4. Sibon I, Sommer P, Lamasiere JM, Bonnet J. Lysyl oxidase deficiency: a new cause of human arterial dissection. Heart. 2005;91(5):e33. 
5. Saw J, Poulter R, Fung A, Wood D, Hamburger J, Buller C. Spontaneous coronary artery dissection in patients with fibromuscular dysplasia: a case series. Circ Cardiovasc Interv. 2012;5(1):134-7.

6. Kar S, Shah PK. Acute coronary syndrome caused by coronary artery dissection mimicking acute plaque rupture. Ver Cardiovasc Med. 2001;2(4):215-9.

7. Virmani R, Forman MB, Robinowitz M, McAllister HA Jr. Coronary artery dissections. Cardiol Clin. 1984;2(4):633-46.

8. Azam MN, Roberts DH, Logan WF. Spontaneous coronary artery dissection associated with oral contraceptive use. Int J Cardiol. 1995;48(2):195-8.
9. Arnold JR, West NE, van Gaal WJ, Karamitsos TD, Banning AP. The role of intravascular ultrasound in the management of spontaneous coronary artery dissection. Cardiovasc Ultrasound. 2008;6:24.

10. Butler R, Webster MW, Davies G, Kerr A, Bass N, Armstrong $\mathrm{G}$, et al. Spontaneous dissection of native coronary arteries. Heart. 2005;91(2):223-4.

11. Buys EM, Suttorp MJ, Morshuis WJ, Plokker HW. Extension of a spontaneous coronary artery dissection due to thrombolytic therapy. Cathet Cardiovasc Diagn. 1994;33(2):157-60.

12. Silva EOA, Furini FR, Mota FM, Lima VC. Dissecção coronária espontânea. Rev Bras Cardiol Invasiva. 2011;19(1):90-2. 